\title{
Measurement of Local Pulse Wave Velocity on Aorta for Noninvasive Diagnosis of Arteriosclerosis
}

\author{
Ryoji Murata $^{1)}$, Hiroshi Kanai ${ }^{1)}$, Noriyoshi Chubachi' ${ }^{1)}$, and Yoshiro Koiwa ${ }^{2)}$ \\ 1) Department of Electrical Engineering, Faculty of Engineering, Tohoku University \\ Aramaki-aza-Aoba, Aoba-ku, Sendai 980-77, Japan \\ 2) First Department of Internal Medicine, Tohoku University School of Medicine \\ Seiryo-machi 2-1, Aoba-ku, Sendai 980-77, Japan
}

\begin{abstract}
Small vibrations are simultaneously measured at two adjacent points on the aortic wall near the aortic valve by electronically alternating the directions of the ultrasonic beam. The transit delay time of the pulse wave between these two points is determined from two vibration signals obtained by this alternation beam method. The local pulse wave velocity at several millimeters on the aorta is precisely obtained by dividing the distance between these points by the resultant transit delay time. These local acoustic properties of the blood vessel will be very useful especially for noninvasive diagnosis of the early stage of arteriosclerosis.
\end{abstract}

Keywords- Arteriosclerosis, Aorta, Pulse Wave Velocity, Ultrasonic Doppler Method

\section{INTRODUCTION}

Measurement of pulse wave velocity (PWV) is known to be very useful for diagnosis of arteriosclerosis. In the standard method, the PWV is obtained from the difference in arrival time of pressure waves propagating from the heart to the femoral artery by using microphones. In this method, the measurable points are limited to where the aorta exists near the skin surface and the distance between such points is long, i.e., several hundred millimeters. Thus, only the average PWV is obtained between these distant points in the method.

In the early stage of arteriosclerosis, fibrous spots with several millimeters in diameter are scattered on the surface of the artery. After growth of these spots, the arterial wall becomes homogeneously hard in the final stage of arteriosclerosis. It is important especially for early diagnosis, therefore, to measure the local hardness of the arterial wall.

This paper proposes a new method to measure local PWV, which is an index of the hardness in the range of several millimeters on the aortic wall, by using the ultrasonic diagnosis equipment.

\section{Principle}

Pulse wave is pressure wave generated by rhythmical ejection of blood from the left ventricle due to systaltic motion of the heart. The PWV $c_{0}$ is given by Meons-Korteweg as follows:

$$
c_{0}=\sqrt{\frac{E h}{2 r \rho}},
$$

where $E, h, r$, and $\rho$ denote Young's modulus, the thickness of blood vessel's wall, the radius of blood vessel, and the density of blood, respectively. Since Meons-Korteweg equation includes Young's modulus $E$, the PWV $c_{0}$ corresponds to the elasticity of the blood vessel very well.
Therefore, measurement of the PWV is very useful for diagnosis of arteriosclerosis.

For measurement of local PWV at several millimeters, it is necessary to simultaneously measure small vibrations $v_{A}(t)$ and $v_{B}(t)$ at two adjacent points $\mathrm{A}$ and $\mathrm{B}$ on the aortic wall near the heart by using an ultrasonic probe. For the aortic wall near the heart, however, their vibrations are small in amplitude (about $\pm 100 \mu \mathrm{m}$, up to at least several hundred hertz) and are superimposed on the motion with large amplitude (about $\pm 10 \mathrm{~mm}$ several hertz) due to the heart beat. To overcome this problem we have proposed a method to estimate the vibration velocity $v(t)$ of an object moving with large amplitude. In this method, the velocity $v(t)$ of the object is estimated by

$$
\widehat{v}\left(t+\frac{\Delta t}{2}\right)=c \frac{\theta_{t+\Delta t}-\theta_{t}}{2 \omega_{0} \Delta t}
$$

where $c, \theta_{t}, \omega_{0}$, and $\Delta t$ denote sound velocity in water, the phase of the reflective ultrasonic wave at a time $t$, the angular frequency of the incident ultrasonic wave, and the time interval between a pulse received at a time $t$ and the successive received pulse, respectively [1-2]. This equation enables us to measure the small vibration $v_{A}(t)$ and $v_{B}(t)$ at the two points $\mathrm{A}$ and $\mathrm{B}$ on the aortic wall.

In this measurement, the employed ultrasonic probe is a sector-type electronic scan probe, by which the ultrasonic beam is transmitted in one of the 128 directions. Each of these directions is designated by the number from 0 to 127 in the ultrasonic diagnosis equipment. By utilizing this principle, we made the circuit, by which the numbers $N_{A}$ and $N_{B}$ corresponding to the two directions of points $A$ and $B$ are controlled synchronously with the transmitted trigger pulse. Therefore, by controlling the two numbers for every transmitted interval $\Delta T$, the beam is alternately transmitted in two different directions using one ultrasonic probe as shown in Fig. 1. The two small vibrations $v_{A}(t)$ and $v_{B}(t)$ are obtained from the ultrasonic signals reflected at points $\mathrm{A}$ and $\mathrm{B}$ using Eq. (2) as shown in Fig. 2.

To measure the local PWV from obtained vibration signals $v_{A}(t)$ and $v_{B}(t)$, the transit delay time $\tau_{A B}$ of the pulse wave which propagates from $A$ to $B$ is determined. Thus, the local PWV $c_{0}$ is estimated from the transit time $\tau_{A B}$ and the distance $d_{A B}$ between these points as follows:

$$
c_{0}=\frac{d_{A B}}{\tau_{A B}}
$$

where the distance $d_{A B}$ is measured on B-mode image 
in the ultrasonic diagnosis equipment using its distancemeasurement function.

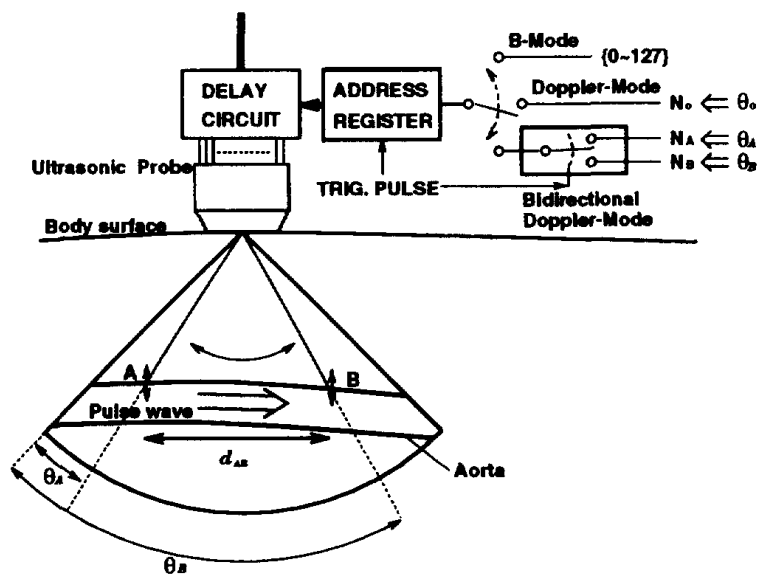

Fig. 1. Measurement of the velocity of the pulse wave which propagates on the silicone tube.

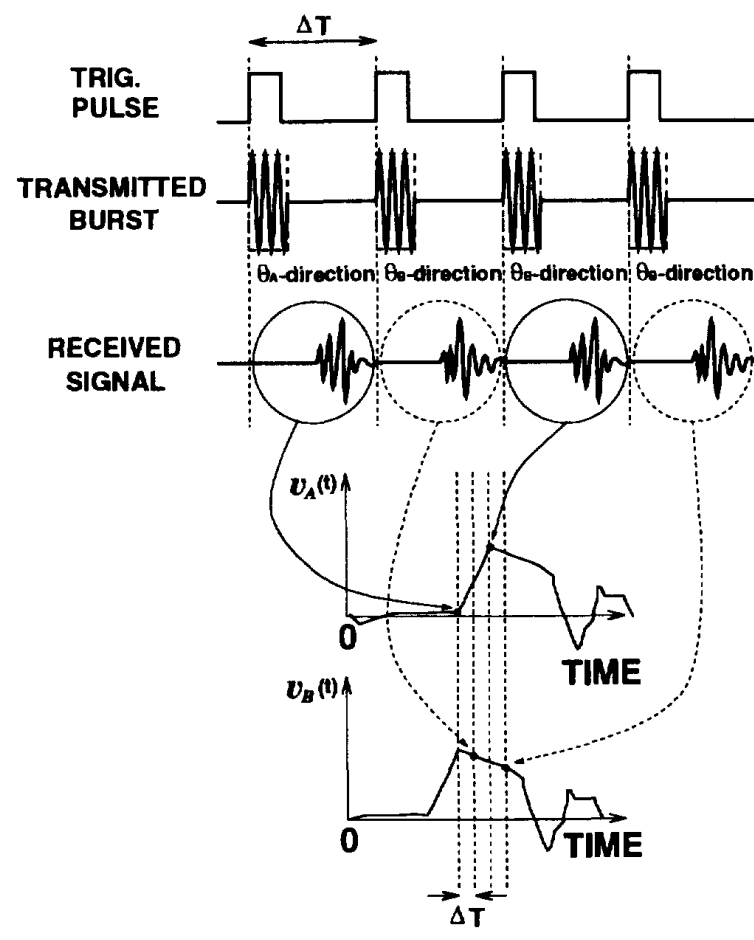

Fig. 2. An illustration explaining the method for obtaining small vibration signals from the received ultrasonic signals, reflected at the two points.

III. In vivo MEASUREMENT OF LOCAL PWV ON AORTA By using this equipment and the circuit we made, the following in vivo experiments of measurement of the local PWV are performed for the aorta in a normal male of age 23. Measurement points are on the aortic wall near the heart and the wall of the abdominal aorta.
ECG
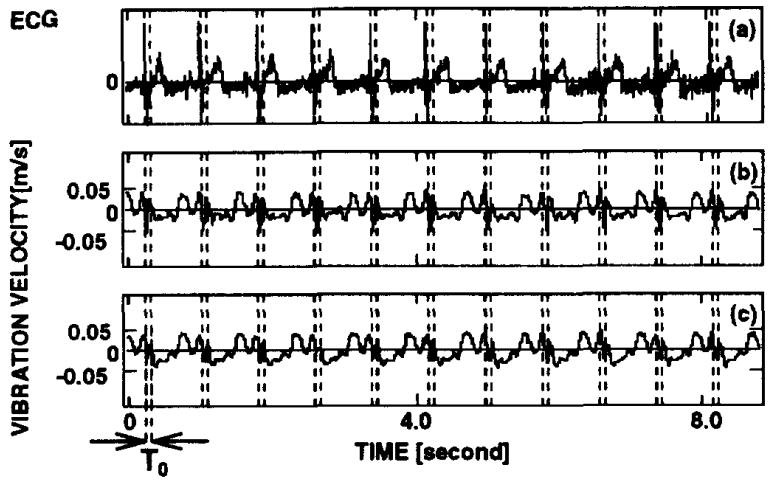

Fig. 3. Simultaneously measured small vibration signals at the two points $A$ and $B$ on the aortic wall near the heart. (a) Electrocardiogram(ECG). (b) Small vibration signal at the point $A: v_{A}(t)$. (c) Small vibration signal at the point $B: v_{B}(t)$.

The small vibrations $v_{A}(t)$ and $v_{B}(t)$ at two points $A$ and $B$ on the aortic wall near the heart are accurately measured as shown in Fig. 3 for the twelve beat periods. The distance $d_{A B}$ between these points is $9.1 \mathrm{~mm}$. The transit time $\tau_{A B}$ from point $A$ to point $B$ is determined from $v_{A}(t)$ and $v_{B}(t)$ in the period $T_{0}$ of $100 \mathrm{~ms}$ in length, which correspond to the ejection period of these twelve heart beats, and $\tau_{A B}$ is $2.44 \mathrm{~ms}$. Thus, the local PWV is $3.7 \mathrm{~m} / \mathrm{s}$ from $\mathrm{Eq}$. (3).

The small vibrations $v_{A}(t)$ and $v_{B}(t)$ are also measured at two points $A$ and $B$ on the wall on the abdominal aorta for nine beat periods from same person. The distance $d_{A B}$ between these points is $7.7 \mathrm{~mm}$. The transit time $\tau_{A B}$ from point $A$ to point $B$ is determined by the similar procedure as described above from $v_{A}(t)$ and $v_{B}(t)$ in the period $T_{0}$ of $200 \mathrm{~ms}$ in length, and $\tau_{A B}$ is $1.85 \mathrm{~ms}$. Thus, the local PWV is $4.2 \mathrm{~m} / \mathrm{s}$.

\section{CONCLUSION}

We proposed a method to obtain the local pulse wave velocity by measuring small vibrations at two adjacent points simultaneously with an ultrasonic probe. This measurement of the local PWV is considered to be effective especially in noninvasive diagnoses of the early stage arteriosclerosis.

\section{REFERENCES}

[1] H. Kanai, H. Satoh, K. Hirose, and N. Chubachi, "A New Method for Measuring Small Local Vibrations in the Heart Using Ultrasound," IEEE Trans. on Biomedical Eng., Vol. 40, pp. 1233-1242, 1993.

[2] K. Kawabe, H. Kanai, and N. Chubachi, "Accuracy Evaluation in Ultrasonic-Doppler-based Measurement of Small Vibrations for Acoustical Diagnosis of the Aortic Wall," Elect. Letters, Vol. 29, pp. 915-916, 1993. 